\title{
Digital Deformable Model Simulating Active Contours ${ }^{\star}$
}

\author{
François de Vieilleville ${ }^{1}$ and Jacques-Olivier Lachaud ${ }^{2}$ \\ Laboratoire de Mathématiques, UMR CNRS 5127 \\ Université de Savoie, 73776 Le-Bourget-du-Lac, France \\ francois.de-vieilleville@univ-savoie.fr, \\ jacques-olivier.lachaud@univ-savoie.fr
}

\begin{abstract}
Deformable models are continuous energy-minimizing techniques that have been successfully applied to image segmentation and tracking since twenty years. This paper defines a novel purely digital deformable model (DDM), whose internal energy is based on the minimum length polygon (MLP). We prove that our combinatorial regularization term has "convex" properties: any local descent on the energy leads to a global optimum. Similarly to the continuous case where the optimum is a straight segment, our DDM stops on a digital straight segment. The DDM shares also the same behaviour as its continuous counterpart on images.
\end{abstract}

\section{Introduction}

Since the pioneering works of Kass et al. 14, deformable models or active contour models have been extremely studied over the past 20 years. They have many applications in image segmentation, tracking and computer vision. They combine in a single framework two terms, one expressing the fit to data (image energy), the other describing shape priors and acting as a regularizer (internal energy). Furthermore, as noted by many authors, the parameter balancing the two terms acts as scale factor, providing a very natural multiscale analysis of images. Deformable models [15], Mumford-Shah approximation [20], geometric or geodesic active contours and other levelset variants [18., are classical variational formulation (i.e. continuous) of such techniques. Energy-minimization for image segmentation can also be expressed in a discrete setting: structural split and merge [8], weighted graph with cut optimization [3], irregular and combinatorial pyramids [12, Markov fields and stochastic processes [10].

Continuous variational problems induce partial differential equations which are solved iteratively. They are most often bound to get stuck in local minima, except in specific cases 761, sometimes with a loss of generality regarding the energies. On the other hand, in several discrete settings, optimal solutions can sometimes be found or better approached 312. However, it should be noted that the first-order regularization term — the length — is generally very coarsely

\footnotetext{
` Partially funded by ANR project FOGRIMMI (ANR-06-MDCA-008-06).
} 
approximated as the number of digital steps. Furthermore, as far as we know, there is no discrete setting that approaches an optimum for gradient-based image energies. Noticing this limitation, Boykov and Kolmogorov [2] have proposed to enrich the neighborhood graph to get finer area estimators - in a way similar in spirit to chamfer distances - but their approach is for now limited to an 8-neighborhood, and seems costly in practice. The work presented here shares the same objective, but it is based on discrete geometry tools.

There are several attemps to define digital analogs of continuous deformable models, migrating processes [9], discrete deformable boundaries, digital curvature flow [13. However, none of them are able to mimick correctly continuous models. They do not solve the same variational problem, even asymptotically with finer and finer grids.

We present here a digital deformable model which incorporates in a digital way the length regularization term. The length estimation is based on an adaptation of the minimum length polygon (e.g., see [17]). It is defined as the shortest euclidean path within a one pixel wide band centered on an open simple digital 4connected path. As in the case of active contour, this length constraint energy is a good regularizer since it defines a kind of convex energy. Optimization heuristics lead to the global optimum. Moreover our deformable model has a behaviour on images which is very close to the one of the original continuous active contour (with first-order regularization).

The paper is organized as follows. We first introduce some remarks and properties on minimum length polygons (Section 2). We then define our digital model and proves the above-mentioned properties (Section 3). Some experiments illustrate the use of our DDM on synthetic images, in particular the impact of the internal energy is shown (Section 4). Eventually, we conclude on the benefits of the proposed approach and propose some future works.

\section{MLP Algorithm and Its Properties}

A 4-connected path in the discrete plane is a sequence of digital points, such that any two consecutive points are 4-neighbors. We restrict our study to simple paths, i.e. paths such that all points are pairwise distinct. Such paths are conveniently described with a Freeman word. The points of a path may be embedded as unit squares, forming a grid continuum in the terminology of Sloboda and Zat́ko [24]. We call $\mathcal{C}$ the set of simple digital paths, and $\mathcal{C}(A, B)$ the subset of $\mathcal{C}$ composed of the paths starting at a point $A$ and ending at a point $B$.

Choosing an orientation (counterclockwise in the remaining of the paper) an inner and outer boundary can be defined. As illustrated on Fig. 1, the inner boundary is the frontier to the left (denoted by $\partial I$ ) and the outer boundary is the frontier to the right (denoted by $\partial O$ ). We are interested in computing the shortest Euclidean path starting and ending at the centroids of the two extremities of the open digital path while staying inside $\partial I$ and $\partial O$ (see Fig. 1). We may notice that the inner and outer boundary may not be simple 4-connected path. This shortest path is very close to the minimum length polygon (MLP), 


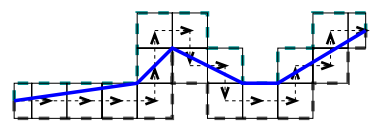

Fig. 1. Portion of a digital contour whose Freeman code word is EEEENNESESEENENE. In terms of concave and convex turns, given the CCW orientation, the contour is read as $(000+0--+-+0+-+-)$. Its inner and outer boundary are drawn with dashed lines. These 4 -connected paths are not necessarily simple. The thick line is the shortest Euclidean path starting and ending at its endpoints.

except that we have two constrained points. We will say therefore say that we compute the constrained MLP, CMLP for short.

Minimum length polygons have a long history [192217]. It was shown that MLPs are unique even for arbitrary constraint polygons. Furthermore, the MLP vertices are either positive turns of $\partial I$ or negative turns of $\partial O$. Moreover there exists an application between the set of negative turns of $\partial I$ and the set of positive turns of $\partial O$. This application just translate the negative vertices of $\partial I$ by one of the following vectors according to the quadrant of the turn: $(1,1)$, $(-1,1),(-1,-1),(1,-1)$. As a consequence, the digital MLP can be computed from $\partial I$. In the case of elements of $\mathcal{C}$, the preceding rule applies except for the first and last vertices of the MLP, which are imposed.

Klette and Yip [17. (reported also in [16]) have proposed a linear time algorithm which seems to fail at finding the proper MLP (see Appendix A).

A correct linear time algorithm could be achieved for instance with computational geometry shortest path algorithms (e.g. see [11]), but would be tough to implement. Since our main objective is related to digital deformable models and not to MLP computation, we have slightly adapted the algorithm of Klette and Yip to compute a CMLP, in a time that may not be optimal in some cases. Certainly an optimal MLP would be an interesting work in itself, and may be a perspective to this work. Let us first introduce a property regarding the elements of $\mathcal{C}$ with a $C M L P$ of minimal length.

Lemma 1. There exists $C \in \mathcal{C}(A, B)$ such that $C M L P(C)=[A B]$. Furthermore, for any $C^{\prime} \in \mathcal{C}(A, B)$, the length of $C M L P\left(C^{\prime}\right)$ is no smaller than the length of the segment $[A B]$.

It is enough to remark that the grid-intersection digitization of the Euclidean segment $[A B]$ is in $\mathcal{C}(A, B)$. Of course there exists other 4-connected paths whose $C M L P$ equals $[A B]$.

In the remaining of the paper, we call $C_{i}$ the points associated to the path $C$ and $P_{j}$ the vertices of the $C M L P$. Let us now consider the constrained minimum length polygon of $C$, denoted by $C M L P(C)$, which is described as a list of pairwise distinct vertices $P_{0} P_{1} \ldots P_{n} P_{n+1}$ with $A=P_{0}$ and $B=P_{n+1}$. Assuming that $n \geq 1$ and that there exists $1 \leq i \leq n$, such that $\operatorname{det}\left(\overrightarrow{P_{i-1} P_{i}}, \overrightarrow{P_{i} P_{i+1}}\right) \neq 0$, $C M L P(C)$ is not the Euclidean segment $[A B]$. According to Sloboda [23], a 
vertex such that $\operatorname{det}\left(\overrightarrow{P_{i-1} P_{i}}, \overrightarrow{P_{i} P_{i+1}}\right)>0$ is called convex and is always a positive point of $\partial I$, similarly, a vertex such that $\operatorname{det}\left(\overrightarrow{P_{i-1} P_{i}}, \overrightarrow{P_{i} P_{i+1}}\right)<0$ is concave and is always a negative point of $\partial O$.

As explained earlier on we are interested in the deformation of constrained open digital contours. Let us now elaborate on our digital deformable model and the associated energy based on the CMLP.

\section{A Digital Deformable Model}

\subsection{Digital Formulation of a Deformable Model}

In 14] Kass et al. proposed the active contour model, which describes a curve whose evolution is monitored by a minimization scheme balancing two terms, an internal regularizing energy and an external energy expressing the fit to data. Let $v$ be a parametrization of the curve embodying the active contour geometry:

$$
\begin{gathered}
v=[0,1] \rightarrow \mathbb{R}^{2} \\
v(s)=\left(\begin{array}{l}
x(s) \\
y(s)
\end{array}\right)
\end{gathered}
$$

with its energy function being defined as:

$$
E_{D M}(v)=\int_{0}^{1}\left(E_{\text {int }}\left(v(s), v^{\prime}(s), v^{\prime \prime}(s)\right)+E_{\text {image }}(v(s))\right) d s .
$$

This formulation allows to define various internal energies based on the position of the curve, its first and second derivative. The internal energy is most commonly found as follow, with $\alpha$ and $\beta$ being parameters that drive the behaviour of the model.

$$
E_{\text {int }}\left(v(s), v^{\prime}(s), v^{\prime \prime}(s)\right)=\left(\alpha(s)\left|v^{\prime}(s)\right|^{2}+\beta(s)\left|v^{\prime \prime}(s)\right|^{2}\right) / 2
$$

Considering that the sought curve is a minimum for $E_{D M}$, its Euler-Lagrange equations induce differential equations whose discretization leads to the usual formulation of active contours.

We propose here a digital vision of the same minimization problem. The geometry of our digital analog is a digital curve, a 4-connected path $\Gamma$. Moreover, we do not consider curvature regularization in this paper, that is we consider $\beta$ equal to zero. As noticed by many authors (see for instance the geometric active contours of [4]), the internal energy is in fact the length of the curve. Our digital analog to $E_{\text {int }}$ is therefore defined as the length of the CMLP of $\Gamma$, that is the sum of the Euclidean length of its edges. Thus our internal energy becomes:

$$
E_{\text {int }}^{D}(\Gamma)=\alpha \mathcal{L}_{2}(C M L P(\Gamma)) ! 1
$$

$\overline{{ }^{1} \mathcal{L}_{2}(C M L P}(\Gamma)$ is to be read as the sum of the Euclidean lengths of the edges of the polygon defined by the CMLP of $\Gamma$. 
The image energy term is defined as a sum over the digital path and is positive everywhere:

$$
E_{\text {image }}^{D}(\Gamma)=\sum_{c \in \Gamma}(\max (\|\nabla I\|)-\|\nabla I(c)\|) .
$$

Our digital combinatorial minimization problem becomes:

$$
\Gamma^{*}=\arg \min _{\Gamma \in \mathcal{C}(A, B)} E_{D M}^{D}(\Gamma), \text { where } E_{D M}^{D}(\Gamma)=E_{\text {int }}^{D}(\Gamma)+E_{\text {image }}^{D}(\Gamma) .
$$

Let us remark that in the case of the defined energies, geodesic active contours provide a way of efficiently finding a global minimum for this problem [7]. However our model allows more general energies such as region energy terms which do not fit in the previously cited approach.

\subsection{Features and Elementary Deformations}

Once an energy is defined for the space of admissible shapes, a set of elementary transitions must be provided. Enough transitions or deformations must be defined so that this space is connected.

We propose several elementary modifications for digital contours, which we call 'flips', 'bumps' and 'flats', see Fig. 2 for an illustration of some elementary deformations. A digital contour is a 4-connected path and is equivalently defined as an ordered list of turns, either toward the interior (denoted "+") or the exterior (denoted "-") or straight ahead moves (denoted "0"). Since they are elements of $\mathcal{C}$, no contour has three consecutive "+" or "-". All proposed deformations preserve this property. We say that an operation is valid if and only if the digital contour is still simple after the operation. Of course all operations are reversible.

- Outside corners, that is $(-+-),(-+0),(0+-),(0+0)$ can be respectively "flipped" into $(0-0),(0-+),(+-0),(+-+)$. This operation is denoted by Flip ${ }^{+}$.

- Inside corners, that is $(0-0),(0-+),(+-0),(+-+)$ can be respectively "flipped" into $(-+-),(-+0),(0+-),(0+0)$. This operation is denoted by Flip ${ }^{-}$, it is the inverse of Flip ${ }^{+}$.

- Inside or outside bumps, that is $(--)$ or $(++)$ can be flatened to () , this is a valid operation. This operation is denoted by Flat.

- Flat inside parts, that is $(--),(-0),(00)$ can be respectively bumped inside to $(0--0),(0--+),(+--+)$. This operation is denoted by Bump ${ }^{-}$.

- Flat outside parts, that is $(++),(+0),(00)$ can be respectively bumped outside to $(0++0),(0++-),(-++-)$. This operation is denoted by Bump ${ }^{+}$.

Remark that some deformations may turn an element of $\mathcal{C}$ into a self-crossing 4 -connected path, it is of course not a valid operation in such cases. We can now define operations on specific contour points. They are related to the convexity of their corresponding vertex on the CMLP. 


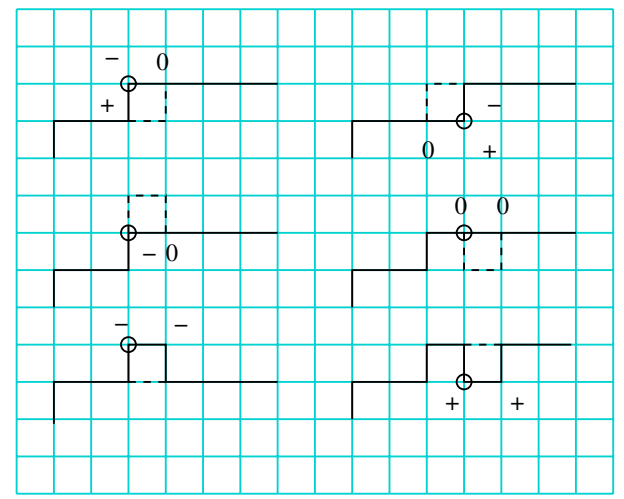

Fig. 2. Example of some local deformations used by the digital deformable model, solid line for $\Gamma$ and dashed line for $\Gamma$ after the local deformation, the points where the deformation is applied are circled. From left to right and top to bottom : flip of an inside corner, flip of an outside corner, inside bump of a flat part, outside bump of a flat part, flat of an inside bump and flat of an outside bump.

Definition 1. Given a convex vertex of the CMLP, it is associated either to one outside corner on $\partial I$ or to one outside bump on $\partial I$. We call convex deformation the Flip ${ }^{+}$of this outside corner or the Flat of this outside bump.

Definition 2. Given a concave vertex of the CMLP, it is associated either to one inside corner on $\partial O$ or to one inside bump on $\partial O$. We call concave deformation the Flip ${ }^{-}$of this inside corner or the Flat of this inside bump.

As we have explained earlier on there exists at least one element of $\mathcal{C}(A, B)$ with minimal energy. Our aim is now to show that despite the fact that some deformations are not valid, there always exists at least one valid convex deformation on a convex vertex or a concave deformation on a concave vertex. For this purpose, we introduce three lemmas:

Lemma 2. On the digital plane, every simple 4-connected path composed of only two Freeman letters and containing a positive or negative corner, say $C_{i}$, cannot also visit the nearby digital point $F \operatorname{lip}\left(C_{i}\right)$.

Proof. Let us consider a positive vertex given by the Freeman code $N E$ with its corresponding points $P_{0}(x, y), P_{1}(x, y+1)$ and $P_{2}(x+1, y+1)$. The corresponding flipped point $\operatorname{Flip}\left(P_{1}\right)$ is $(x+1, y)$. In order the path to visit Flip $\left(P_{1}\right)$ after visiting $P_{2}$, it must contain a $S$ letter in the Freeman code word since its $y$ coordinate is lower than that of $P_{2}$. If it were to visit it before $P_{0}$ then it should contain a $W$ letter since its $x$ coordinate is greater than that of $P_{0}$.

Lemma 3. On the digital plane every simple 4-connected path $C$ defined with 3 or more Freeman letters is such that whenever $a \ldots \bar{a}$ is a factor of $C$ then the associated turns of $a \ldots \bar{a}$ contain either $\left(+0^{*}+\right)$ or $\left(-0^{*}-\right)$. 
Proof. It is enough to consider the associated points to the $a$ letter and $\bar{a}$ letter, without loss of generality, let $a=E$ and $\bar{a}=O, P_{a_{1}}=(x, y)$ and $P_{a_{2}}=$ $(x+1, y), P_{\bar{a}_{1}}=\left(x^{\prime}, y^{\prime}\right)$ and $P_{\bar{a}_{2}}=\left(x^{\prime}-1, y^{\prime}\right) . C$ being simple we have necessarily $(x+1, y) \neq\left(x^{\prime}-1, y^{\prime}\right)$ and $(x, y) \neq\left(x^{\prime}-1, y^{\prime}\right)$ and $(x, y) \neq\left(x^{\prime}, y^{\prime}\right)$.

Considering the minimal bounding box containing the factor $a \ldots \bar{a}$, its height is greater than one, therefore its right side intersects $a \ldots \bar{a}$ with a $N^{m}$ or $S^{m}$ move, with $m \geq 1$, that is an $E N^{m} O$ move or a $E S^{m} O$ move.

Lemma 4. On the digital plane, if $C$ is a simple 4-connected path containing an outside or inside corner, that is three points $C_{i-1}, C_{i}$ and $C_{i+1}$ such that $\operatorname{sign}\left(C_{i}\right)$ is positive or negative, and visiting also the point $F$ lip $\left(C_{i}\right)$, then it contains either $-0^{*}-$ or $+0^{*}+$ between $C_{i}$ and $F \operatorname{lip}\left(C_{i}\right)$.

Proof. Without loss of generality, we choose a $N E$ corner, that is an outside corner. Let $C_{i-1}=(x, y), C_{i}=(x, y+1), C_{i+1}=(x+1, y+1)$ be the points embodying it on the digital plane. Since $C$ is simple and has to visit the point $F \operatorname{Fip}\left(C_{i}\right)=(x+1, y)=C_{j}$, with $j>i$, let us consider two cases:

- either $C_{j-1}=(x+2, y)$ and $C_{j+1}=(x+1, y-1)$, that is a $O S$ corner;

- or $C_{j-1}=(x+1, y-1)$ and $C_{j+1}=(x+2, y)$, that is a $N E$ corner.

In the first case Lemma 3 shows that there exists either $-0^{*}-$ or $+0^{*}+$ between $E$ and $O$.

In the second case we see that $C_{i+1}=(x+1, y+1)$ and $C_{j-1}=(x+1, y-1)$ entails that there is at least two $S$ letters between the two $N E$ corners. Thus by Lemma 3 there exists either $-0^{*}-$ or $+0^{*}+$ between $S$ and $N$.

We may now prove that any digital contour whose CMLP is not a segment is "deformable".

Proposition 1. Let $C \in \mathcal{C}(A, B)$ being finite, if $C M L P(C) \neq[A B]$ there exists at least one valid convex deformation on a convex vertex or one valid concave deformation on a concave vertex of $C M L P(C)$.

Proof. We prove it by contradiction. Let us consider that $C M L P(C) \neq[A B]$ and that there do not exist any valid convex deformation on a convex vertex or a valid concave deformation on a concave vertex of $C M L P(C)$.

First, all deformations on $C M L P(C)$ are necessarily flips on outside or inside turns since the flat deformation is always in $\mathcal{C}(A, B)$ and is by definition always a valid convex deformation on a convex vertex or a valid concave deformation on a concave vertex.

$C$ is thus constituted of inside turns, outside turns and straight parts, and at least one of the inside or outside turn is associated to a CMLP vertex. Since by hypothesis those corners are not allowed to be flipped, the contour visits also the points associated to each corner flip.

In the case where $C$ is only constituted of two Freeman letters, Lemma 2 concludes, since convex deformations can be applied to convex vertices and concave deformation can be applied to concave vertices. 
Let us now consider that $C$ has at least 3 Freeman letters and, without loss of generality, the case of a positive vertex of the CMLP. By definition it is associated to an outside corner in $C$, let $C_{i}$ be the corresponding point associated to the positive turn. From Lemma 4. $C$ contains at least $\left(+0^{m}+\right)$ with $m \geq 1$ between $C_{i}$ and Flip $\left(C_{i}\right)$. Since bumps would entail valid deformations, we have further that $m \geq 1$. As a result, the $\left(+0^{m}+\right)$ quadrant change would entail at least one more outside corner. Since they are points of quadrant changes, a vertex of the CMLP is associated to it. Applying this reasoning recursively leads to an infinite number of points on $C$. As we are on a finite simple 4-connected path, this raises a contradiction. Similarly, the same reasoning applies on an inside vertex.

\subsection{Two Greedy Deterministic Optimization Schemes}

We define two simple deterministic greedy optimization scheme. Algorithm 1 selects the deformation which brings the lowest energy from all the valid deformations that can be applied to the digital deformable model (DDM for short). Algorithm 2, even greedier, picks the first deformation that diminishes the energy of the DDM.

Function Greedy1( In $\Gamma$, Out ) : boolean ;

Input: $\Gamma$ : a Digital Deformable Model

Output: $\Gamma^{\prime}$ : when returning true, elementary deformation of $\Gamma$ with $E_{D M}^{D}\left(\Gamma^{\prime}\right)<E_{D M}^{D}(\Gamma)$, otherwise $\Gamma^{\prime}=\Gamma$ and it is a local minimum.

Data: $Q$ : Queue of (Deformation, double) ;

$E_{0} \leftarrow E_{D M}^{D}(\Gamma)$;

foreach valid Deformation d on $\Gamma$ do

$\Gamma$.applyDeformation $(d)$;

$Q \cdot \operatorname{push} \_b a c k\left(d, E_{D M}^{D}(\Gamma)\right)$;

$\Gamma$.revertLastDeformation();

end

$\left(d, E_{1}\right)=$ SelectDeformationWithLowestEnergy $(Q)$;

$\Gamma^{\prime} \leftarrow \Gamma$;

if $E_{1}<E_{0}$ then $\Gamma^{\prime}$.applyDeformation $(d)$;

return $E_{1}<E_{0}$;

Algorithm 1. Greedy1 algorithm: extracts the deformation that brings the lowest energy among all possibles ones

\subsection{Properties}

We will now show a remarkable property of our discrete internal energy. It is similar to a convex energy in the continuous case, in the sense that any local descent leads to one of the optimal solutions.

The following theorem proves that we always reach a configuration of minimal energy when choosing at each step an arbitrary valid deformation which decreases the energy. This induces that both Greedy1 and Greedy2 algorithms lead to one of the optimal solution. 
Function Greedy2( In $\Gamma$, Out ) : boolean ;

Input: $\Gamma$ : a Digital Deformable Model

Output: $\Gamma^{\prime}$ : when returning true, elementary deformation of $\Gamma$ with $E_{0} \leftarrow E_{D M}^{D}(\Gamma)$

$E_{D M}^{D}\left(\Gamma^{\prime}\right)<E_{D M}^{D}(\Gamma)$, otherwise $\Gamma^{\prime}=\Gamma$ and it is a local minimum.

foreach valid Deformation d on $\Gamma$ do

$\Gamma$.applyDeformation $(d)$;

if $E_{D M}^{D}(\Gamma)<E_{0}$ then $\Gamma^{\prime} \leftarrow \Gamma$;

return true;

I.revertLastDeformation();

end

$\Gamma^{\prime} \leftarrow \Gamma$;

return false;

Algorithm 2. Greedy2 algorithm : extracts the first valid deformation that decreases the energy

Theorem 1. Let $C \in \mathcal{C}(A, B)$ being finite, choosing at each step any one of the valid convex or concave deformation leads to $C M L P(C)=[A B]$ after a finite number of steps.

Proof. There are 2 cases, either:

- $C M L P(C)=[A B]$, and we have reached the global minimum of energy, by definition of a shortest path.

- $C M L P(C) \neq[A B]$ and in this case we have $C M L P(C)=P_{0} P_{1} \ldots P_{n} P_{n+1}$ with $A=P_{0}$ and $B=P_{n+1}$ with $n \geq 1$ and there exist at least one vertex of the CMLP which is convex or concave, that is there exists $i$ such that either $\operatorname{det}\left(\overrightarrow{P_{i-1} P_{i}}, \overrightarrow{P_{i} P_{i+1}}\right)>0$ or $\operatorname{det}\left(\overrightarrow{P_{i-1} P_{i}}, \overrightarrow{P_{i} P_{i+1}}\right)<0$.

In the second case, let us first suppose that $P_{i}$ is associated to an outside or inside bump on $C$. Without loss of generality we consider the convex case, that is $\operatorname{det}\left(\overrightarrow{P_{i-1} P_{i}}, \overrightarrow{P_{i} P_{i+1}}\right)>0$ and an outside bump. By construction, $\left[P_{i-1} P_{i}\right]$ and $\left[P_{i} P_{i+1}\right]$ intersect the sides of the unit square centered on $P_{i}$ on two distinct points $I_{1}$ and $I_{2}$. The triangle $I_{1} P_{i} I_{2}$ is within the unit square centered on $P_{i}$ and of non zero area. The length of $\left[I_{1} I_{2}\right]$ is obviously shorter than that of $\left[P_{i-1} P_{i}\right]+$ $\left[P_{i} P_{i+1}\right]$. As a result, the flat operation on the outside bump entails that the transformed CMLP has a length no greater than $P_{0} \ldots P_{i-1} I_{1} I_{2} P_{i+1} \ldots P_{n+1}$. The energy has then strictly diminished.

Let us now suppose that $P_{i}$ is associated to an outside or inside corner on $C$. From Prop. 1 if the corner associated to $P_{i}$ cannot be flipped, then there exists at least one inside or outside corner on $C$ associated to a convex or concave vertex of the CMLP. Without loss of generality, let it be an outside corner associated to a convex vertex of the CMLP. By construction, $\left[P_{i-1} P_{i}\right]$ and $\left[P_{i} P_{i+1}\right]$ intersect the sides of the unit square centered on $P_{i}$ on two points $I_{1}$ and $I_{2}$. The triangle $I_{1} P_{i} I_{2}$ is within the unit square centered on $P_{i}$ and of non zero area. Following the same reasoning as earlier, the transformed CMLP brings a lower energy. 

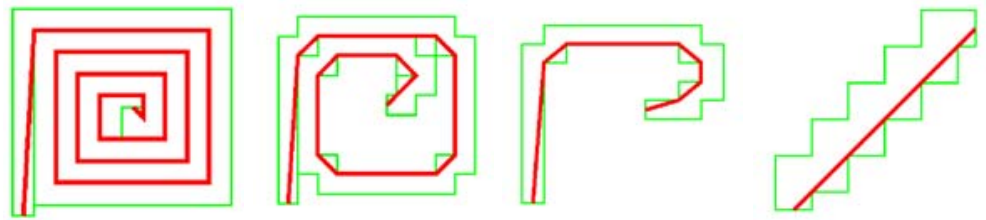

Fig. 3. Example of the minimization process on a spiral using the Greedy1 algorithm. Only the length penalisation term is used in this process. In green the inner and outer boundary are drawn, in red the CMLP is drawn. From left to right: Initialisation of the DDM. Result of the minimisation energy process after 50 iterations. Result of the minimisation energy process after 100 iterations. End of the process after 139 iterations, which is also a global minimum.
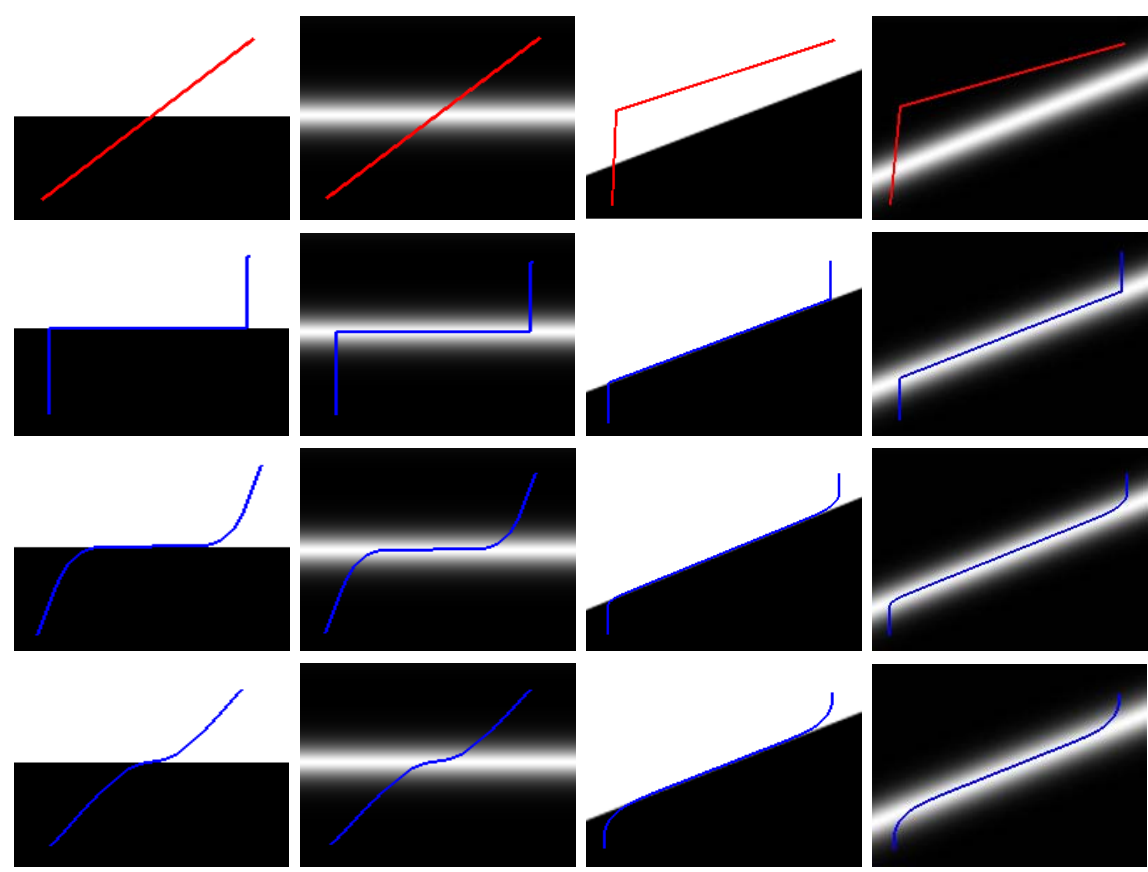

Fig. 4. Example of the minimization process using the Greedy1 algorithm. The gradient is computed with the Canny-Deriche method with scale coefficient 0.2. The input image represents a half-plane. (First row) Initialisation of the DDM. (Second row) Results of the minimisation process, the $\alpha$ coefficient used is equal to 0 . (Third row) Results with $\alpha=200$. (Fifth row) Results with $\alpha=300$. 
Note that it is possible to force the minimization process to choose the gridintersection digitization of $[A B]$ by selecting the path with lowest energy and maximizing the number of positive turns. This slight modification reduces the number of optimal solutions to exactly one, as in the continuous case.

\section{Experimental Evaluation}

We here show some preliminary results of our DDM. Figure 3 shows that the internal energy, i.e. the regularization term, is indeed a kind of convex energy: the spiral is unwinded to the digital straight segment by Greedy1 algorithm. Figure 4 illustrates the balance between internal energy and gradient-based image energy. Again, the observed behaviour matches the expected bahaviour of classical snakes: the lower the length penalisation term, the more the DDM sticks
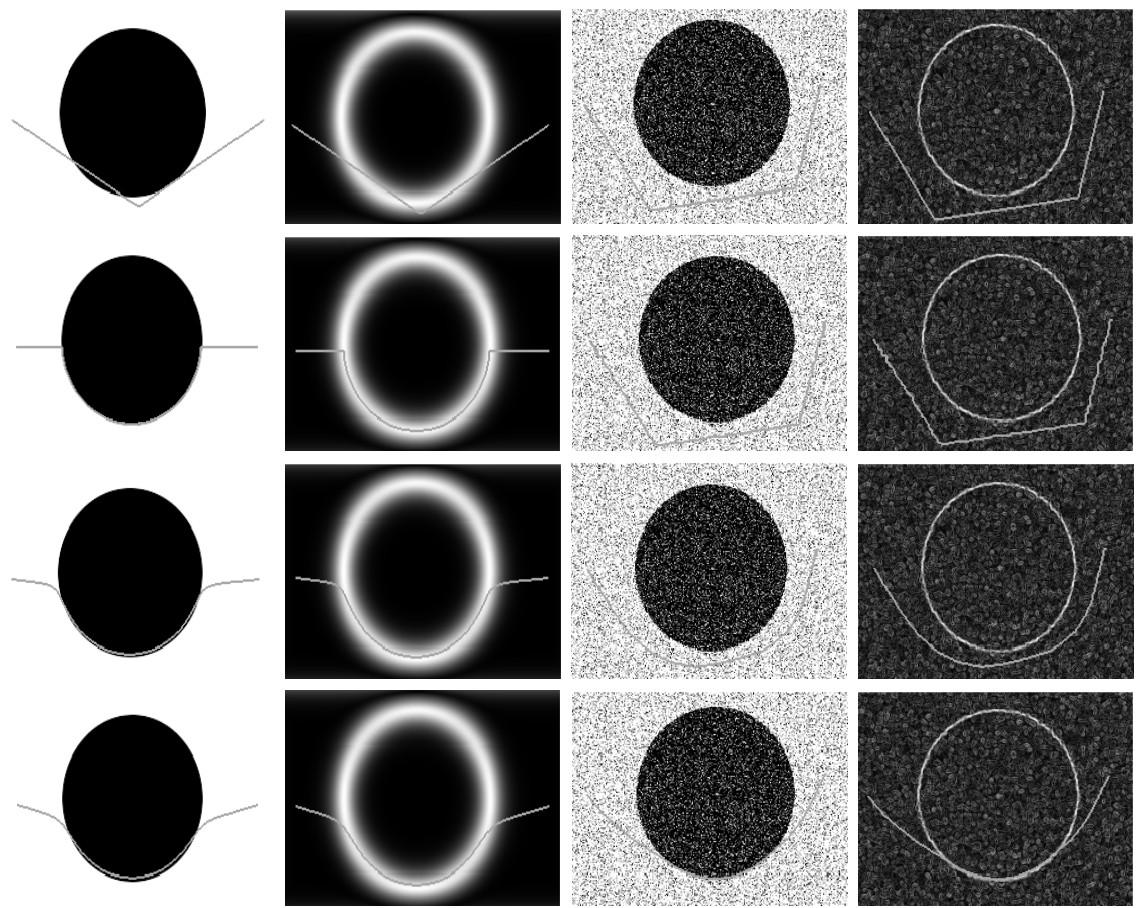

Fig. 5. Example of the minimization process using the Greedy2 algorithm. In order to test our DDM in extreme cases, the gradient is computed with the Canny-Deriche method with scale coefficient 0.2 for the ellipse image without noise, and coefficient 2.0 for the noisy ellipse image. The gradient field is thus very perturbated in the latter case. (First row) Initialisation of the DDM. (Second row) Results of the minimization process, the $\alpha$ coefficient is equal to 0 . (Third row) Results with $\alpha=800$ for the circle without noise and $\alpha=4000$ for the noisy circle. (Fourth row) Results with $\alpha=1600$ for the circle without noise and $\alpha=8000$ for the noisy circle. 
to points having strong gradients. Finally, we tested our model on images degradated with a strong Gaussian noise, as displayed on Fig. 5. In an extremely noisy environment, a very strong regularizing term brings effectively the DDM to escape local image energy minima so as to fit more consistent gradient information that is further away (bottom right of figure).

\section{Conclusion}

In this paper we have proposed a Digital Deformable Model based using the Minimum Length Polygon as length penalisation energy. We have given theoretical results that back up our claim that it is a digital analog to the continuous variational formulation of standard active contours. The regularizing term has indeed "convex-like" properties, and the optimum is the digitization of straight line segment. Moreover experimental results on images have shown the proposed DDM behave as expected, even with very basic optimization heuristics.

Future works will focus on the use of this DDM with other optimization scheme, such as stochastic processes (simulated annealing is appealing) or combinatorial optimization algorithms (our model seems related to submodular functions [21]). We plan also to embed it in topological maps, in order to propose a model for deforming partitions in dimension two. This would lead to an interesting digital analog to multiphase levelset segmentation methods or Mumford-Shah approximation.

\section{References}

1. Ardon, R., Cohen, L.D.: Fast constrained surface extraction by minimal paths. International Journal on Computer Vision 69(1), 127-136 (2006)

2. Boykov, Y., Kolmogorov, V.: Computing geodesics and minimal surfaces via graph cuts. In: Proc. Int. Cof. Comput. Vis (ICCV 2003), Nice, France, November 2003, vol. 1, pp. 26-33 (2003)

3. Boykov, Y., Veksler, O., Zabih, R.: Fast approximate energy minimization via graph cuts. IEEE Transactions on Pattern Analysis and Machine Intelligence 23(11), 1222-1239 (2001)

4. Caselles, V., Catte, F., Coll, T., Dibos, F.: A geometric model for active contours. Numerische Mathematik 66, 1-31 (1993)

5. Caselles, V., Kimmel, R., Sapiro, G., Sbert, C.: Minimal surfaces based object segmentation. IEEE Trans. Pattern Anal. Mach. Intell. 19(4), 394-398 (1997)

6. Chan, T.F., Vese, L.A.: Active contours without edges. IEEE Trans. on Image Processing 10(2), 266-277 (2001)

7. Cohen, L.D., Kimmel, R.: Global minimum for active contour models: a minimal path approach. Int. Journal of Computer Vision 24(1), 57-78 (1997)

8. Dupas, A., Damiand, G.: First results for 3D image segmentation with topological map. In: Coeurjolly, D., Sivignon, I., Tougne, L., Dupont, F. (eds.) DGCI 2008. LNCS, vol. 4992, pp. 507-518. Springer, Heidelberg (2008) 
9. Fejes, S., Rosenfeld, A.: Discrete active models and applications. Pattern Recognition 30(5), 817-835 (1997)

10. Geman, S., Geman, D.: Stochastic relaxation, Gibbs distributions, and the Bayesian restoration of images, pp. 564-584. Morgan Kaufmann Publishers Inc., San Francisco (1987)

11. Guibas, L.J., Hershberger, J.: Optimal shortest path queries in a simple polygon. In: SCG 1987: Proceedings of the third annual symposium on Computational geometry, pp. 50-63. ACM, New York (1987)

12. Guigues, L., Le Men, H., Cocquerez, J.-P.: Scale-sets image analysis. In: Int. Conf. on Image Processing (ICIP 2003), vol. 2, pp. 45-48. IEEE, Los Alamitos (2003)

13. Imiya, A., Saito, M., Tatara, K., Nakamura, K.: Digital curvature flow and its applications for skeletonization. Journal of Mathematical Imaging and Vision 18, 55-68 (2003)

14. Kass, M., Witkin, A., Terzopoulos, D.: Snakes: Active contour models. International Journal of Computer Vision, 321-331 (1988)

15. Kass, M., Witkin, A., Terzopoulos, D.: Snakes: Active contour models. International Journal of Computer Vision 1(4), 321-331 (1988)

16. Klette, R., Rosenfeld, A.: Digital Geometry - Geometric Methods for Digital Picture Analysis. Morgan Kaufmann, San Francisco (2004)

17. Klette, R., Yip, B.: The length of digital curves. Machine Graphics Vision 9(3), 673-703 (2000) (Also research report CITR-TR-54, University of Auckland, NZ. 1999)

18. Malladi, R., Sethian, J.A., Vemuri, B.C.: Shape Modelling with Front Propagation: A Level Set Approach. IEEE Trans. on Pattern Analysis and Machine Intelligence $17(2), 158-174$ (1995)

19. Montanari, U.: A note on minimal length polygonal approximation to a digitized contour. Commun. ACM 13(1), 41-47 (1970)

20. Mumford, D., Shah, J.: Optimal approximations by piecewise smooth functions and associated variational problems. Comm. Pure Appl. Math. 42, 577-684 (1989)

21. Schrijver, A.: Combinatorial optimization: polyhedra and efficiency. Springer, Heidelberg (2004)

22. Sloboda, F., Stoer, J.: On piecewise linear approximation of planar jordan curves. J. Comput. Appl. Math. 55(3), 369-383 (1994)

23. Sloboda, F., Stoer, J.: On piecewise linear approximation of planar jordan curves. Journal of Computational and Applied Mathematics 55, 369-383 (1994)

24. Sloboda, F., Zat́ko, B.: On one-dimensional grid continua in r2. Technical report, Institute of Control Theory and Robotics, Slovak Academy of Sciences, Bratislava, Slovakia (1996)

\section{A Detailed Steps of Klette and Yip Algorithm}

Klette and Yip 17. (reported also in 16]) have proposed a linear time algorithm which fails sometimes at finding the proper MLP, as shown on Fig. 6. This is explained in where several steps of the algorithm are reproduced on Table 1 and match those illustrated on the previous figure. 

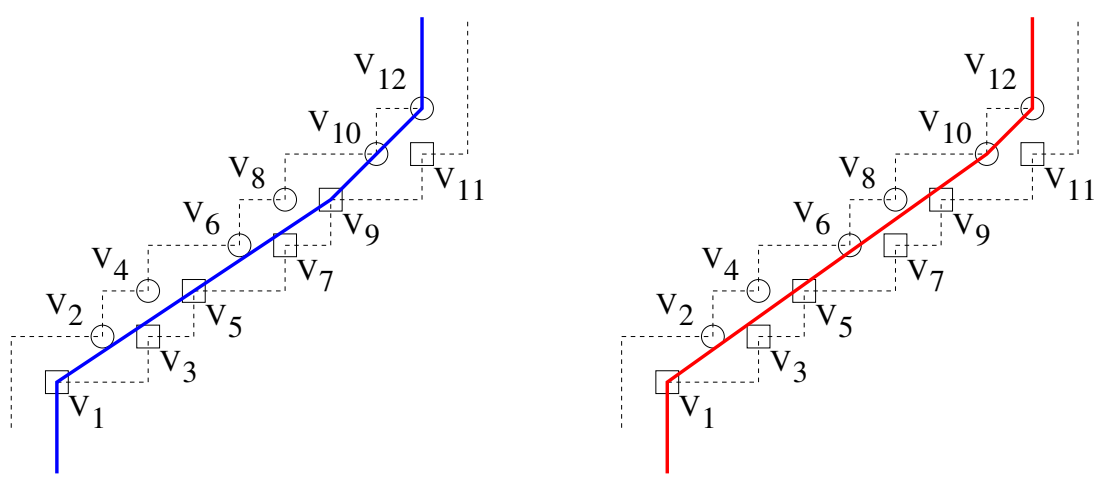

Fig. 6. Portion of a boundary where the algorithm of 17 fails at electing the correct Minimum Length Polygon vertices. The positive points are circled and the negative points are boxed. (Left) The MLP elected by Klette's algorithm, vertices are $\left(v_{1}, v_{5}, v_{9}, v_{12}\right)$. (Right) the correct portion of MLP, whose vertices are $\left(v_{1}, v_{10}, v_{12}\right)$.

Table 1. Several steps of the algorithm of Fig. [6 are reproduced here. The input list is $\left(v_{1}, v_{2}, v_{3}, v_{4}, v_{5}, v_{6}, v_{7}, v_{8}, v_{9}, v_{10}, v_{11}\right)$. Where $v_{1}$ is the first MLP vertex considered.

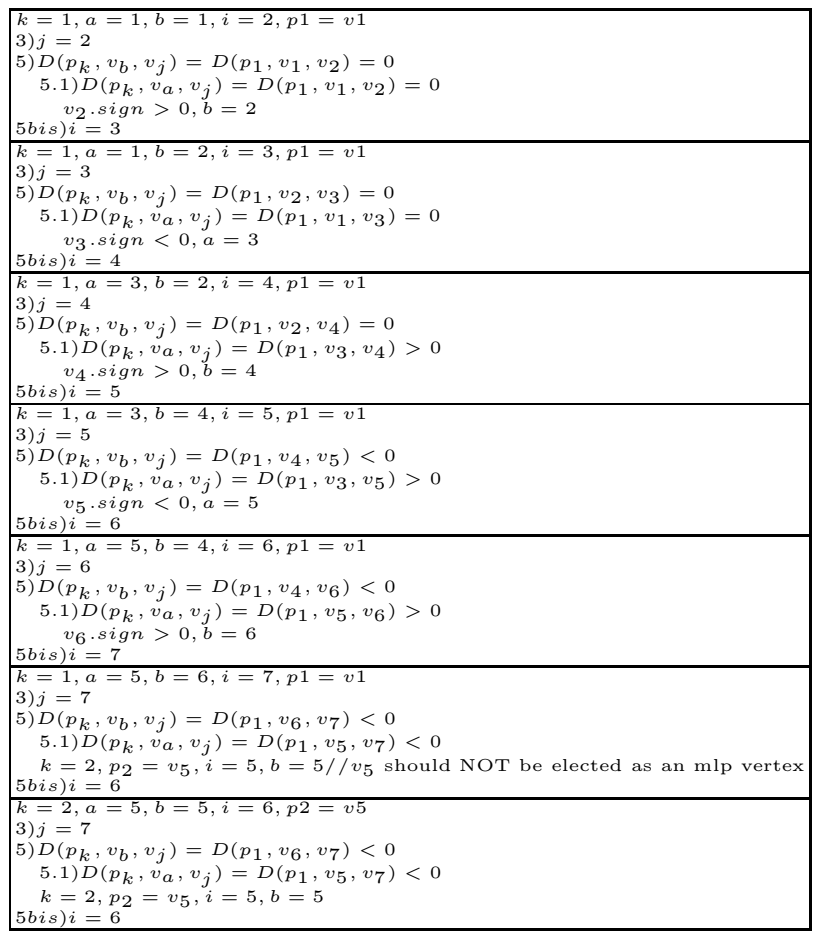

